\title{
CRYPTOSPORIDIUM HOMINIS PREDOMINANCE AMONG SYMPTOMATIC EGYPTIAN CHILDREN
}

\author{
By \\ SAMIR A.TAHA ${ }^{1 *}$, ZEIN ABD AL AAL ${ }^{1}$, NESREEN S. SALEH ${ }^{2}$ \\ AND AYMAN A. EL-BADRY ${ }^{3}$
}

Department of Zoology, Faculty of Science, Zagazig University ${ }^{1}$, Department of

Zoology, Faculty of Science, Benghazi University, Libya ${ }^{2}$ and Department of Medical

Parasitology, Faculty of Medicine, Cairo University ${ }^{3}$, Cairo, Egypt

( ${ }^{*}$ Correspondence: sameertaha@hotmail.com, Mobile 01003811559)

\section{Abstract}

Cryptosporidiosis is a worldwide gastrointestinal disease caused by a protozoan parasite; Cryptosporidium. This study determined the prevalence of Cryptosporidium spp. in fecal samples collected from children, and assessed the association between Cryptosporidium in stool with the patient's age, gender and with seasonality. Fecal samples were collected from 176 children (1-12 years) attending gastrointestinal outpatients' clinics in Aboul-Reesh Cairo University Pediatrics Hospital, Egypt. The samples were examined microscopically and by nested polymerase chain reaction (nPCR) assay targeting Cryptosporidium oocyst wall protein (COWP) gene. The nPCR products were digested by restriction enzyme Rsa I. The obtained fragments were resolved by electrophoresis.PCR was more sensitive than microscopic examination in estimating Cryptosporidium prevalence. Cryptosporidium oocysts were found in 4.5\% (8/176) of samples. Cryptosporidium DNA was in $11.9 \%$ (21/176). C. hominis (17 cases or $81 \%$ ) was the predominant species among symptomatic children, peaking in the summer and a small rise in autumn. This study demonstrated that age, gender and season might shape the prevalence of such protozoa in a given population.

Keywords: Cryptosporidium, children, COWP gene, PCR-RFLP, nPCR, prevalence

\section{Introduction}

Cryptosporidium infects a wide range of vertebrates, including humans, recognized as an important gastrointestinal human pathogen (Caccio et al, 2005). Cryptosporidium spp. are well adapted to zoonotic transmission usually occurs through a direct fecaloral route and through oocysts contaminating water or food (Smith et al, 2007). Cryptosporidium spp. can cause a wide spectrum of symptoms, from severe life-threatening diarrhea and vomiting in immunocompromised patients to asymptomatic and selflimiting infection in immunocompetent individuals (Omrani et al, 2015). The parasite can be of zoonotic or anthroponotic origin, and has been implicated in diarrhea outbreaks in different parts of the world (Xiao 2010; Adamu et al, 2014). Cryptosporidium is listed as a neglected disease by the World Health Organization, largely due to a lack of studies in developing countries, but lastly it gained increasing attention (Savioli et al, 2006).
Cryptosporidium has 24 valid species and more than 44 genotypes infecting many vertebrates, including animals and humans, which differ significantly in their molecular signatures (Cama et al, 2008; Xiao 2010; Ryan et al, 2014). There are many intestinal and gastric Cryptosporidium species that are capable of establishing infection in immunocompetent and immunocompromised individuals (Akiyoshi et al, 2003; Fayer et al, 2010; Elwin et al, 2012). Among these species, Cryptosporidium parvum (which also infects ruminants and other animals) and $C$. hominis (almost exclusively found in humans) are the most common species in clinical human infections (Sulaiman et al, 2005).

Using the molecular diagnostics has greatly enhanced the detection of infection and transmission of Cryptosporidium (Xiao, 2010). PCR) has been frequently used for identifying the species and genotypes of Cryptosporidium due to its higher sensitivity than microscopic examination (Stark et al, 2008; Salyer et al, 2012). 
The study aimed to determine the prevalence of Cryptosporidium spp. in fecal samples collected from children, and to assess the association between Cryptosporidium in stools with patient's age, gender and with seasonality.

\section{Materials and Methods}

The present cross-sectional study was conducted from August 2016 to July 2017 throughout the 4 different seasons. A total of 176 fecal samples were collected from symptomatic Egyptian children from Great Cairo, attending GIT outpatient clinics at Aboul-Reesh hospital Cairo. This study has been approved by the ethical committee of Zagazig University and has been performed in accordance with the ethical standards as laid down in the 1964 Declaration of Helsinki and its later amendments or with comparable ethical standards. Families of all children were informed verbally about the purpose of the study, and the collection of samples was performed after obtaining consent. Participation in the study was optional.

Microscopic examination: A single fecal sample from each child was collected and exami-ned after acid-fast staining for Cryptosporidium oocysts (El-Naggar et al, 2006).

Sample processing for molecular analyses: Molecular analyses were done at the lab of Molecular Medical Parasitology (LMMP), Department of Medical Parasitology, Faculty of Medicine, Cairo University, Egypt. Each collected fecal sample was subjected to Cryptosporidium copro-DNA amplification by nPCR and specification of Cryptosporidium species using PCR-RFLP.

Total genomic DNA was extracted from the stool samples using the Favor Stool DNA Spin Columns Isolation Mini Kit (Favorgen Biotech Corporation ping-Tung 908, Taiwan) with a modification in the form of prolongation of incubation at the temperature 95 for 1 hour after thermal shock (cycling of deep freezing in liquid nitrogen for $5 \mathrm{~min}$ and immediately transferred into a water bath at $95^{\circ} \mathrm{C}$ for $5 \mathrm{~min}$; repeated for 5 cycles), then the extracted DNA was as- sessed for concentration and purity using Qubit 2.0 Fluor meter and then stored at $20^{\circ} \mathrm{C}$.

The nPCR was carried out for all the collected fecal samples $(n=176)$ in two consecutive reactions targeting the COWP gene. The first reaction a fragment of 769 base pair (bp) was amplified using a set of primers (Pedraza-Díaz et al, 2001). The produced fragment was used as a template in a second reaction in whicha553bp was amplified using inner primers set (Spano et al, 1997). Each reaction was done using a PCR mix: $12.5 \mu 1$ PCR Master Mix [Thermo Scientific, UK), $1 \mu 1$ of $200 \mathrm{nmol} / \mathrm{l}$ of each forward and reverse primer, $2.5 \mu \mathrm{l}$ of template DNA, $0.1 \mu 1$ Taq polymerase $(5 \mathrm{U} / \mu \mathrm{l})$ [product Thermo Scientific]and $7.9 \mu 1$ of sterile distilled water to complete a total volume of $25 \mu 1$. Reactions were performed in a gradient thermal cycler (thermo-cycler, Biometra; Applied Biosystems, California, USA) after adjusting the thermal profile to initial denaturation at $95^{\circ} \mathrm{C}$ for $4 \mathrm{~min}$, followed by 30 cycles of amplification, each consisting of denaturation at $94^{\circ} \mathrm{C}$ for 60 seconds, annealing at $65^{\circ} \mathrm{C}$ for 60 second and extension at $72^{\circ} \mathrm{C}$ for 60 second. Final elongation was performed at $72^{\circ} \mathrm{C}$ for $10 \mathrm{~min}$. The secondround PCR was identical to the first-round PCR except for denaturation at $94^{\circ} \mathrm{C}$ for 50 second, annealing at $54^{\circ} \mathrm{C}$ for 30 second, and extension at $72^{\circ} \mathrm{C}$ for 50 second. The amplified PCR products were separated by electrophoresis on $2 \%$ agarose gel and further visualized under a UV trans-illuminator after being stained with ethidium bromide (Tab. 1).

Cryptosporidium genotyping by PCRRestriction fragment length polymorphism (PCR-RFLP): A volume of $10 \mu \mathrm{l}$ of nPCR product was digested in a mixture consisting of $1 \mu 1$ RsaI (No. ER1121, Thermo Scientific) $2 \mu \mathrm{l}$ green buffer, and $17 \mu \mathrm{l}$ nuclease-free water to reach a volume of $30 \mu$. The mix was mixed gently followed by spinning down for a few seconds and then incubated at $37^{\circ} \mathrm{C}$ for $5 \mathrm{~min}$. Cryptosporidium geno- 
types were detected by using electrophoresis in $3.2 \%$ typing-grade agarose gels containing ethidium bromide, and gels were visualized under UV trans-illumination. Digestion pattern of the fragment was different for each characteristic Cryptosporidium species infecting human: $C$. hominis $(34,106,125 \&$ 285bp) and C. parvum (34,106 and 410bp).

Statistical analysis: Data were analysed a chi-square test of association on the infected and non-infected samples for all risk factors. Significance of association was expressed as $P$-value according to the following parameters: $\mathrm{P}>0.05$ : non-significant, $\mathrm{P}<0.05$ : significant. The statistical analyses were conducted using Graph Pad Prism V.5software (Graph Pad Software, Inc., San Diego, California).

\section{Results}

The patients' gender distribution was 92 males \& 84 females. Ages ranged from 1 to 12 years old; 25 were 1 to 2 years, 87 between 3 to 8 years and 64 between 9 to 12 years.

Microscopically the oocysts in children's feces were confirmed morphologically to be those of Cryptosporidium. Oocysts were spherical in shape and had smooth wall. Out of the 176 microscopically examined fecal samples, $8(4.5 \%)$ fecal samples were Cryptosporidium oocyst positive (Tab. 2).

The nPCR analysis that targeted the COWP gene (553bp) identified Cryptosporidium DNA in $11.9 \%(21 / 176)$ of the samples. With respect to gender, there was higher prevalence of infection in samples collected from male children than that from female children (Fig. 1). The higher prevalence was observed for children in early childhood (3-8years) than children in late childhood (9-12 years). No positive cases were found in infants (0-2 years). Also, Cryptosporidium was more frequently in summer and autumn and less in spring and winter (Tab. 3).

Using Chi-Square test of association, there was significant association between being infected with Cryptosporidium and the age, gender and season in which the samples were collected $(\mathrm{P}=0.04)$ using nPCR.

C. hominis pattern was shown $17(81 \%)$ of digested Cryptosporidium DNA, while 4 (19\%) showed C. parvum pattern (Fig. 2).

Table 1: Primers used for the PCR-based genotyping of Cryptosporidium

\begin{tabular}{|c|c|c|c|c|}
\hline Primers & Primer Sequence & Size (bp) & PCRs & Reference \\
\hline BCOWP F & 5' ACCGCTTCTCAACAACCATCTTGTCCTC 3' & \multirow[t]{2}{*}{$769 \mathrm{bp}$} & \multirow[t]{2}{*}{$1^{\text {st }} \mathrm{PCR}$} & \multirow{2}{*}{$\begin{array}{l}\text { Pedraza-Díaz } \\
\text { et al, } 2001\end{array}$} \\
\hline BCOWP R & AATGTAAACCC 3' & & & \\
\hline & 'G 3' & \multirow[t]{2}{*}{$553 \mathrm{bp}$} & \multirow[t]{2}{*}{$2^{\text {st }} \mathrm{PCR}$} & \multirow{2}{*}{$\begin{array}{l}\text { Spano et al, } \\
1997\end{array}$} \\
\hline Cry- 9 & 5'GGACTGAAATACAGGCATTATCTTG3' & & & \\
\hline
\end{tabular}

Table 2: Cryptosporidium spp., in samples $(\mathrm{n}=176)$ using stained microscopic samples and $\mathrm{nPCR}$.

\begin{tabular}{|c|c|c|c|}
\hline Technique & No. examined & No. infected & Prevalence (\%) \\
\hline Microscopic examination & 176 & 8 & 4.5 \\
\hline nPCRs & 176 & 21 & 11.9 \\
\hline
\end{tabular}

Table 3: Cryptosporidium in different children gender, age and in different seasons by microscopy and nPCR.

\begin{tabular}{|c|c|c|c|c|c|c|c|}
\hline \multirow{2}{*}{\multicolumn{2}{|c|}{ Variants }} & \multirow{2}{*}{$\begin{array}{c}\text { No. } \\
\text { examined }\end{array}$} & \multicolumn{2}{|c|}{ Microscopy } & \multicolumn{2}{|c|}{ nPCR } & \multirow[t]{2}{*}{$P$ value } \\
\hline & & & No. & $(\%)$ & No. & $(\%)$ & \\
\hline \multirow{2}{*}{ Sex } & Male & 92 & 6 & 6.52 & 16 & 17.39 & \multirow{2}{*}{$0.045(*)$} \\
\hline & Female & 84 & 2 & 2.38 & 5 & 5.95 & \\
\hline \multirow{3}{*}{ Age } & Infant (1-2 years) & 25 & 0 & 0 & 0 & 0 & \multirow{3}{*}{$0.045(*)$} \\
\hline & Early childhood (3-8 years) & 87 & 5 & 5.74 & 17 & 19.54 & \\
\hline & Late childhood (9-12 years) & 64 & 3 & 4.68 & 4 & 6.25 & \\
\hline \multirow{4}{*}{ Season } & Summer & 72 & 5 & 6.94 & 11 & 15.27 & \multirow{4}{*}{$0.045(*)$} \\
\hline & Autumn & 33 & 2 & 6.60 & 6 & 12.12 & \\
\hline & Winter & 21 & 0 & 0 & 1 & 4.76 & \\
\hline & Spring & 50 & 1 & 2 & 3 & 6 & \\
\hline
\end{tabular}




\section{Discussion}

The Cryptosporidium infection prevalence in the Egyptian patients varied significantly from $0 \%$ to $47 \%$ (Youssef et al, 2008).

In the present study, $4.5 \%$ prevalence of Cryptosporidium spp. was microscopically identified, which was lower than that measured by using the nPCR approach (11.9\%). This agreed with reports in Egyptian children in Cairo. El-Helaly et al. (2012) and ElMatrawy et al. (2017) detected Cryptosporidium oocysts in $2.6 \%, 1.3 \%$, respectively, using microscopy after staining with $\mathrm{AF}$ stain. In contrast, higher prevalence $(32 \%)$ of cryptosporidiosis was reported by AlBraiken et al. (2003) in Saudi Arabia using microscopy. In Egypt, El-Hamshary et al. (2008); El-Settawy and Fathy (2012) reported relatively high prevalence rates in Egyptian children, $21 \%$ \& $18.6 \%$, respectively, using the same technique. These data gave discrepancies in the prevalence of Cryptosporidium, and suggested the need for more accurate and sensitive methods.

In the present study, using nPCR showed that $11.9 \%$ of children had Cryptosporidium infection. This was higher than those of Abd-El-Kader et al. (2012); Sharma et al. (2013) and El-Matrawy et al. (2017) who reported cryptosporidiosis among diarrheic Egyptian children using nPCR to be $4.6 \%$, $3.8 \% \& 6 \%$, respectively. On the other hand, among diarrheic Egyptian children using nPCR El-Hamshary et al. (2008); Abdelrazek et al. (2016); El-Badry et al. (2017) and Salyer et al. (2012) found that the prevalence of Cryptosporidium was relatively higher than that in the present study $(25 \%$, $25 \%, 23.6 \%, \& 32.4 \%$ ) respectively..

The discrepancies in the reported prevalence of other studies and the current one might be attributed to differences in population demographics, environmental, behavioral and socioeconomic factors, as well as differences in timing of sample collection (e.g. summer vs. winter).

Age and gender proved to be risk factors affecting the prevalence of Cryptosporidium in certain populations (Abdelrazek et al, 2016).

In the present study, the Cryptosporidium prevalence was found in early and late childhood, but not in infants. These results agreed with studies conducted by Abdel-Messih et al. (2005); Al-Shamiri et al. (2010); Iqbal et al.(2011) and Abdelrazek et al.(2016). In contrast, El-Helalya et al. (2012) found that the peak prevalence of cryptosporidiosis was in children aged 1-5 years old. All these factors might increase probability of Cryptosporidium detection or exposure to infection (Abdelrazek et al, 2016).

In the present study, Cryptosporidium was more common among males than females, which could be due to a larger sample size of males than females, or might be due to more frequent exposure of male children to gardens and farm animals. This agreed with previous studies (Abd El-Kader et al, 2012; El-Badry et al, 2015; Ibrahim et al, 2016). On the other hand, Yoder et al. (2007) found that most of the reported cases in 2005 occurred among females, and Mathew et al. (2014) also reported higher cryptosporidiosis infection in females $(57.1 \%)$ compared to males (42.9\%). El-Helalya et al. (2012), Abdelrazek et al. (2016) showed that males and females had similar positive rates without significant association. Similarly, Abd El Kader et al. (2012) found that infection rates did not vary with gender.

The present study revealed that the prevalence of Cryptosporidium peaked in summer with, small a rise increase in autumn. These results agreed with El-Badry et al. (2015) who found that Cryptosporidium was more prevalent in summer and a small rise in spring. In contrast, Iqbal et al. (2011) found that the maximum cryptosporidiosis cases were detected in winter.

In the present study, two genotypes, $C$. hominis and $C$. parvum, were detected in stool of the children, with $C$. hominis predominance in all patient groups. Similarly, other studies in Egypt (El-Badry et al, 2015; Abdelrazek et al, 2016; Abdelrazek et al, 
2016; Ghallab et al, 2016; El-Badry et al, 2017), as well as worldwide studies (Bushen et al, 2007; Xiao, 2010) reported C. hominis predominance.

\section{Conclusion}

The conventional microscopic technique used for the diagnosis of Cryptosporidium has a limited capacity and low sensitivity in identifying Cryptosporidium spp. On the other hand, nPCR RFLP analysis of COWP gene fragment proved to be of high sensitivity and was useful for diagnosis and genotyping of Cryptosporidium. The C. hominis was more prevalent in stool samples of symptomatic Egyptian children living in Great Cairo. This suggests that $C$. hominis is of relatively greater risk for humans infections than other species, notably $C$. parvum. The infection rate was higher in male than female children and mainly in early and late childhood. The rate of infection was higher in summer and autumn than in spring and winter months. This study showed that certain risk factors might determine the prevalence of such protozoa in a given population.

Conflict of interest: The authors declare that they neither have conflict of interest nor received fund.

\section{References}

Abd El Kader, MN, Blanco, MA, Ali-Tammam, M, Abd El Ghaffar, R, Osman, A, et al, 2012: Detection of Cryptosporidium parvum and Cryptosporidium hominis in human patients in Cairo, Egypt. Parasitol. Res. 110:161-6.

Abdel-Messih, IA, Wierzba, TF, Abu-Elyazeed, R, Ibrahim, AF, Ahmed, SF, et al, 2005: Diarrhea associated with Cryptosporidium parvum among children of the Nile River Delta in Egypt. J. Trop. Pediatr. 51:154-9.

Abdelrazek, NM, Al-Antably, ASA, Fathy, M M, El-Badry, AA, 2016: Copro-molecular characterization of Cryptosporidium spp. and genotypes among Egyptian children. J. Egypt Soc. Parasitol. 46:385-96.

Adamu, H, Petros, B, Zhang, G, Kassa, H, Amer, S, et al, 2014: Distribution and clinical manifestations of Cryptosporidium species and subtypes in HIV/AIDS patients in Ethiopia. PloS. Negl. Trop. Dis. 8:e283-131.

Al-Braiken, FA, Amin, A, Beeching, NG, Ho- mmel, M, Hart, CA, 2003: Detection of Cryptosporidium amongst diarrheic asymptomatic children in Jeddah, Saudi Arabia. Ann. Trop.

Med. Parasitol. 97:1-5.

Akiyoshi, DE, Dilo, J, Pearson, C, Chapman, S, Tumwine, J, et al, 2003: Characterization of Cryptosporidium meleagridis of human origin passaged through different host species. Infect. Immun. 71:1828-32.

Al-Shamiri, AH, Al-Zubairy, AH, Al-Mamari, RF, 2010: The prevalence of Cryptosporidium spp. in children, Taiz District, Yemen. Iran. J. Parasitol. 5:26-32.

Bushen, OY, Kohli, A, Pinkerton, RC, Dupnik, K, Newman, RD, et al, 2007: Heavy Cryptosporidium infections in children in northeast Brazil: comparison of Cryptosporidium hominis and Cryptosporidium parvum. Trans. R. Soc. Trop. Med. Hyg. 101:378-84.

Caccio, SM, Thompson, RCA, Mc-Lauchlin, J, Smith, HV, 2005: Unraveling Cryptosporidium and Giardia epidemiology. Trends Parasitol. 21:430-7.

Cama, VA, Bern, C, Roberts, J, Cabrera, L, Sterling, CR, et al, 2008: Cryptosporidium species and subtypes and clinical manifestation in children, Peru. Emerg. Infect. Dis. 14:1567-74.

El-Badry, AA, Abdel Aziz, IZ, Shoeib, EY, Ghallab, MMI, 2017: Cryptosporidium genotypes and associated risk factors in a cohort of Egyptian children. Comp. Clin. Pathol. 26:101721.

El-Badry, AA, Al-Antably, ASA, Hassan, A M, Hanafy, NA, Abu-Sarea, EY, 2015: Molecular seasonal, age and gender distributions of Cryptosporidium in diarrhoeic Egyptians: distinct endemicity. Eur. J. Clin. Microbiol. Infect. Dis. 34:2447-53.

El-Hamshary, EM, El-Sayed, HF, Hussein, E M, Soliman, RS, 2008: Comparison of polymerase chain reaction, immunochromatographic assay and staining techniques in diagnosis of cryptosporidiosis. Parasitol United J 1:77-86.

El-Helalya, N, Alyb, M, Attia, S, 2012: Detection of Cryptosporidium infection among children with diarrhea. NY Sci. J. 5:68-76.

El-Matrawy, OM, Hassan, MA, Morsy, SM, Rubio, JM, El-Badry, AA, 2017: Molecular diagnosis of Cryptosporidium spp. versus microscopy in diarrheic patients in Cairo. J. Egypt Soc. Parasitol. 47:303-8.

El-Naggar, SM, El-Bahy, MM, Abd Elaziz, J, 
El-Dardiry, MA, 2006: Detection of protozoal parasites in stools of diarrheic patients using different techniques. J. Egypt. Soc. Parasitol. 36, 1:7-22.

El-Settawy, MA, Fathy, GM, 2012: Evaluation and comparison of PCR, coproantigen ELISA and microscopy for diagnosis of Cryptosporidi$u m$ in human diarrhoeic specimens. J. Am. Sci. 8:1378-85.

Elwin, K, Hadfield, SJ, Robinson, G, Grouch, ND, Chalmers, RM, 2012: Cryptosporidium viatorum sp. among travellers returning to Great Britain from the Indian subcontinent, 20072011. Int. J. Parasitol. 42:675-82.

Fayer, R, Santin, M, Macarisin, D, 2010: Cryptosporidium ubiquitum sp. in animals and humans. Vet. Parasitol. 172:23-32.

Ghallab, MMI, Abdel-Aziz, IZ, Shoeib, EY, El-Badry, AA, 2016: Laboratory utility of coproscopy, copro immunoassays and copro nPCR assay targeting Hsp90 gene for detection of Cryptosporidium in children, Cairo, Egypt. J. Parasitol. Dis. 40:901-5.

Ibrahim, MA, Abdel-Ghany, AE, Abdel-Latef, GK, Abdel-Aziz, SA, Aboelhadid, SM, 2016: Epidemiology and public health significance of Cryptosporidium isolated from cattle, buffaloes, and humans in Egypt. Parasitol. Res. 115:2439-48.

Iqbal, J, Khalid, N, Hira, PR, 2011: Cryptosporidiosis in Kuwaiti children: association of clinical characteristics with Cryptosporidium species and subtypes. J. Med. Microbiol. 60:647-52. Mathew, AO, David, OO, Olubunmi, FI, Mosunmola, OJ, Tiamiyu, AR, Gbenga, AO, 2014: Infection rate of Cryptosporidium parvum among diarrhea children in Ibadan, Oyo State, Nigeria. Sch. J. App. Med. Sci. 2:3127-31.

Omrani, VF, Fallahi, SH, Rostami, A, 2015: Prevalence of intestinal parasite infections and associated clinical symptoms among patients with end-stage renal disease undergoing hemodialysis. Infection 43:537-44.

Pedraza-Díaz, S, Amar, C, Nichols, GL, McLauchlin, J, 2001: Nested polymerase chain reaction for amplification of the Cryptosporidi- um oocyst wall protein gene. Emerg. Infect. Dis. 7:49-56.

Ryan, U, Fayer, R, Xiao, L, 2014: Cryptosporidium species in humans and animals: current understanding and research needs. Parasitology 141, 13:1667-85.

Salyer, SJ, Gillespie, TR, Rwego, IB, Chapman, CA, Goldberg, TL, 2012: Epidemiologyand molecular relationships of Cryptosporidium spp. in people, primates, and livestock from Western Uganda. Negl. Trop. Dis. 6:e1597. DOI: 10. 1371/pntd. 0001597.

Samie, P, Bessong, O, Obi, CL, Sevilleja, JE, Stroup, S, 2006: Cryptosporidium species: preliminary descriptions of the prevalence and genotype distribution among school children and hospital patients in the Venda region, Limpopo Province, South Africa. Exp. Parasitol. 114:31422.

Savioli, L, Smith, H, Thompson, A, 2006: Giardia and Cryptosporidium join the 'Neglected Diseases Initiative'. Trends Parasitol. 22:203-8.

Sharma, P, Sharma, A, Sehgal, R, Malla, N, Khurana, S, 2013: Genetic diversity of Cryptosporidium isolates from patients in North India. Int. J. Infect. Dis. 17:e601-5.

Smith, HV, Caccio, SM, Cook, N, 2007: Cryptosporidium and Giardia as food borne zoonoses. Vet. Parasitol. 149:29-40.

Spano, F, Putignani, L, Naitza, S, Puri, C, Wright, S, Crisanti, A, 1997: Molecular cloning and expression analysis of a Cryptosporidium parvum gene encoding a new member of the thrombospond in family. Mol. Biol. Parasitol. 92:147-62.

Sulaiman, IM, Hira, PR, Zhou, L, Al-Ali, FM, Al-Shelahi, FA, et al, 2005: Unique endemicity of cryptosporidiosis in children in Kuwait. J. Clin. Microbiol. 43:2805-9.

Xiao, L, 2010: Molecular epidemiology of cryptosporidiosis: an update. Exp. Parasitol. 124:809.

Yoder, JS, Beach, MJ, Centers, for Disease Control and Prevention 2007: Giardiasis surveillance:United States, 2003-2005. MMWR Surveill. Summ. 56:1-8

\section{Explanation of figurers}

Fig. 1: Agarose gel electrophoresis for the products of the nPCR targeting COWP gene of Cryptosporidium at 553 bp. Lane1: DNA molecular weight marker100 bp; lane2: negative control; lane3: positive control; lane 4, 6 and 7: negative samples; lane 5 and 8: positive samples.

Fig. 2: Agarose gel electrophoresis showing RFLP products after digestion with Rsa I enzyme endonuclease with Cryptosporidium hominis. Lane1: DNA molecular weight marker100 bp; lane 2 and 3: undigest product of the nested polymerase chain reaction (nPCR) targeting the COWP gene of Cryptosporidium at $553 \mathrm{bp}$; lane 4 and 5: Cryptosporidium hominis genotype digestion product at $34,106,125$ and 285 bps ( 34 band is very small, faint and difficult to see). 


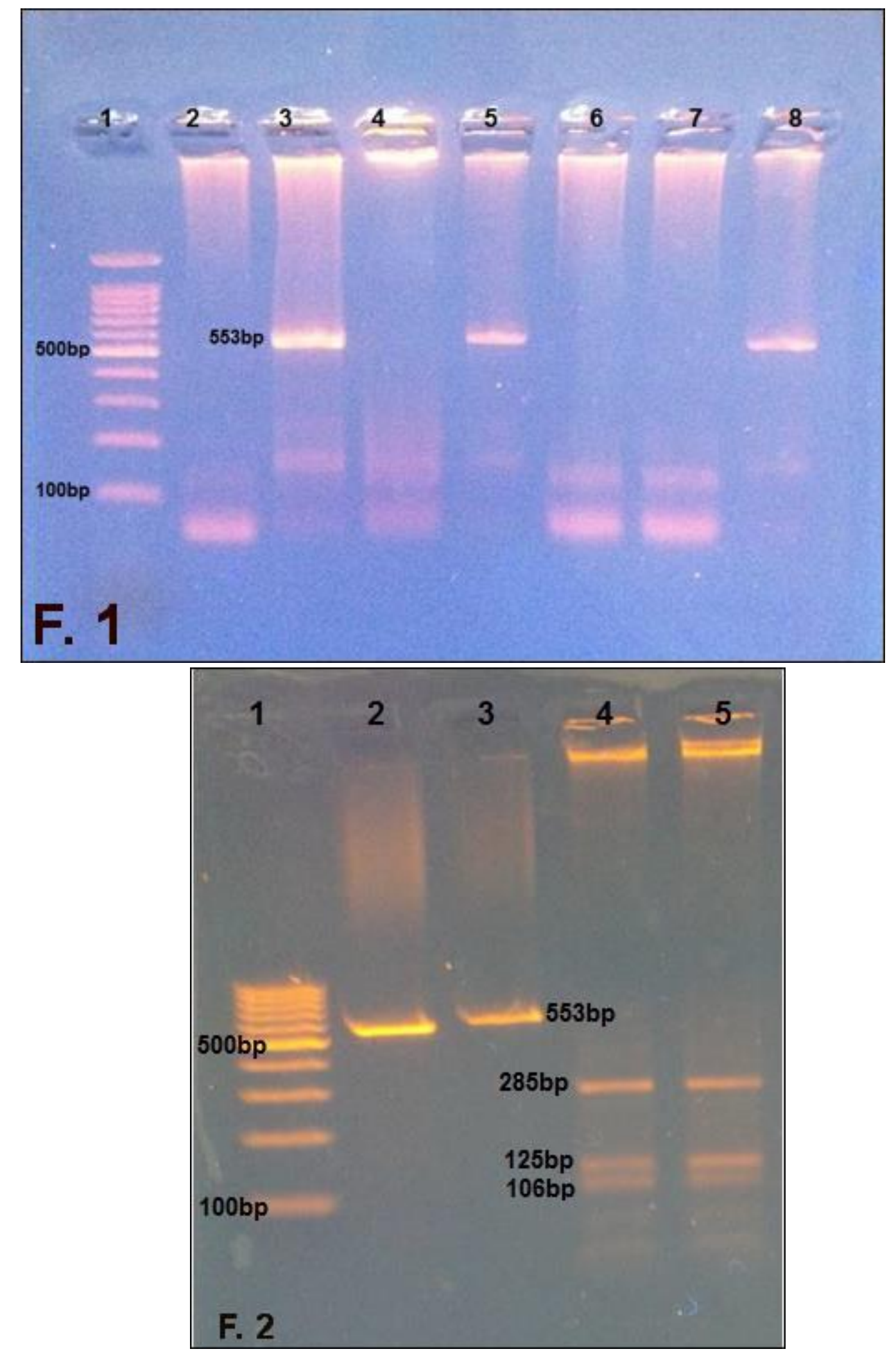

